$\checkmark$ Research Square

\title{
Plasma Neuregulin 1 as a Synaptic Biomarker in Alzheimer's Disease.
}

\section{Agathe Vrillon ( $\square$ agathe.vrillon@aphp.fr)}

Université de Paris

François Mouton-Liger

Université de Paris

Matthieu Martinet

Université de Paris

Emmanuel Cognat

Université de Paris

Claire Hourregue

Université de Paris, Lariboisière Fernand-Widal Hospital, APHP

Julien Dumurgier

Université de Paris, Lariboisière Fernand-Widal Hospital, APHP

Elodie Bouaziz-Amar

Université de Paris

\section{Ann Brinkmalm}

The Salhgrenska Academy at the University of Gothenburg

\section{Kaj Blennow}

The Salhgrenska Academy at the University of Gothenburg

\section{Henrik Zetterberg}

The Salhgrenska Academy at the University of Gothenburg

Jacques Hugon

Université de Paris

Claire Paquet

Université de Paris

\section{Research Article}

Keywords: Alzheimer's disease, NRG1, synaptic pathology, plasma biomarker

Posted Date: February 15th, 2022

DOI: https://doi.org/10.21203/rs.3.rs-1353170/v1 
License: (c) (i) This work is licensed under a Creative Commons Attribution 4.0 International License. Read Full License 


\section{Abstract}

Background:Synaptic dysfunction is an early core feature of Alzheimer's disease (AD), closely associatedwith cognitive symptoms. Neuregulin 1 (NRG1) is a growth and differentiation factor with a key role in the development and maintenance of synaptic transmission. Previous reports have shown that changes in cerebrospinal fluid (CSF) NRG1 concentrationare associated with cognitive status and biomarker evidence of AD pathology. Plasma biomarkers reflecting synaptic impairment would be of great clinical interest.

Objective: To measure plasma NRG1 concentration in AD patientsin comparison with other neurodegenerative disorders and neurological controls (NC)and to study its association with cerebrospinal fluid (CSF) core AD and synaptic biomarkers.

Methods: This retrospective study enrolled 127 participants including patients with AD at mild cognitive impairment stage (AD-MCl, $n=27)$ and at dementia stage $(n=35)$, non-AD dementia ( $n=26, A \beta$-negative), non-AD MCl $(n=19)$ and neurological controls $(n=20)$. Plasma and CSF NRG1, as well as CSF core AD biomarkers ( $A \beta 42 / A \beta 40$ ratio, phospho-tau and total tau), were measured using ELISA. CSF synaptic markers were measured using ELISA for GAP-43 and neurogranin and through immunoprecipitation mass spectrometry for SNAP-25.

Results: Plasma NRG1 concentration was higher in AD-MCI and AD dementia patients compared with neurological controls (respectively $P=0.005$ and $P<0.001$ ). Plasma NRG1 differentiated AD MCI patients from neurological controls with an area under the curve of $88.3 \%$, and $A D$ dementia patients from NC with an area under the curve of $87.3 \%$. Plasma NRG1 correlated with CSF NRG1 ( $\beta=0.372, P=0.0056$, adjusted on age and sex). Plasma NRG1 was associated with AD CSF core biomarkers in the whole cohort and in $A \beta$-positive patients ( $\beta=-0.197-0.423$ ). Plasma NRG1 correlated with CSF GAP-43, neurogranin and SNAP25 ( $\beta=0.278-0.355)$. Plasma NRG1 concentration correlated inversely with MMSE in the whole cohort and in $A \beta$-positive patients (all, $\beta=-0.188, P=0.038 ; A \beta+: \beta=-0.255, P=0.038$ ).

Conclusion: Plasma NRG1 concentration is increased in AD patients and correlates with CSF core AD and synaptic biomarkers, and cognitive status. Thus, plasma NRG1 is a promising non-invasive biomarker to monitor synaptic impairment in AD.

\section{Introduction}

Synaptic impairmentisa core feature of Alzheimer's disease (AD) and one of the earliest detectable changes $(1,2)$.Neuropathological examination has demonstrated that synaptic demise shows higher association to cognitive decline than amyloid plaques load or neurofibrillary tangles pathology $(3,4)$.Positron emission tomography (PET) imaging using synaptic tracers indicates that synaptic density is significantly reduced in the hippocampus in AD patients, especially in its early symptomatic stages $(5,6)$. The evaluation of several synaptic proteins has been achieved in the cerebrospinal fluid (CSF)(7-10).Presynaptic synaptosomal-associated protein 25 (SNAP- 
25),synaptotagmin or(GAP-43) growth-associated protein-43 as well as post-synaptic neurogranin levels are altered in AD CSF and are reliable biomarkers of synaptic impairment, as early as in the preclinical stage of the disease $(11,12)$. In blood, the presynaptic betasynuclein measured using quantitative mass spectrometry could discriminate AD and CJD from controls and other neurodegenerative disorders (13). Other synaptic markers have been explored in blood but so far, due to the existence of peripheral expression or of other factors of variability, there is no validated reliable biomarker of synaptic pathology (14).

Neuregulin 1 (NRG1), a protein encoded by the NRG1 gene, isa member of the epithelial growth factor (EGF) family.Theyconstitute ligands with high affinity for ErbB tyrosine kinase receptors. NRG1 is implicated in many processes during neural development including proliferation of neuronal progenitors, neuron migration and survival, axon guidance, glial development and myelination, as well as synaptogenesis $(15,16)$. In the adult brain, NRG1 is expressed in multiple regions and regulates neurotransmission and synaptic plasticity(17). Membrane-bound, NRG1 requires processing by a protease to initiate release and signaling. Among implicated proteases, NRG1 can undergo cleavage by the $\beta$-site amyloid precursor protein cleaving enzyme 1 (BACE1)at multiple sites(18-20). Proteolytic processing results in the secretion of soluble forms that will further activate ErbB receptors, mainly at the post-synaptic level. NRG1 andits receptor ErbB4 levels have been found altered in human AD brain, both in hippocampus and cortex $(21,22)$.In CSF, two studies including our prior work have reportedmodified NRG1 levels in AD patients compared with controlsand to patients with non-ADrelated cognitive decline $(23,24)$.

The purpose of our study was to investigateplasma NRG1 levels in a cohort of patients with cognitive decline du to $A D$, non- $A D$ related cognitive decline and neurological controls and to assess its association with core AD CSF biomarkers, CSF synaptic markers and cognitive status.

\section{Methods}

\section{Cohort}

A total of 127 patientsfrom the Cognitive Neurology Center, Lariboisière Fernand Widal Hospital, Université de Pariswas retrospectively included in our study. Patients had undergoneCSF biomarkers analysis from 2012 to 2015 including $A \beta 42 / A \beta 40$ ratio, tau phosphorylated on threonine 181 (p-tau) and totaltau (t-tau)measurements, in the context of the diagnostic workup of a cognitive complaint. We had a two-fold clinical and biological approach. Patients were first studied in relation to diagnosis, comprising patients with $\mathrm{AD}$ at the stage of mild cognitive impairment (AD-MCl, $n=27)$ and the stage of dementia $(n=35)$, non-AD related mild cognitive decline (non-AD MCl, $n=19)$, non-ADdementia $(n=26)$ and neurological controls (NC, $n=20$ ). Patients were then dichotomized according to their amyloid status

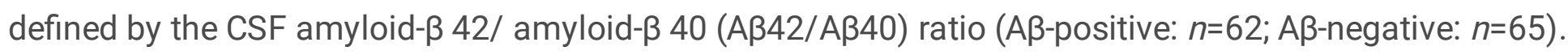

Consensus diagnoses were made by neurologists, geriatricians, neuropsychologists, neuroradiologistsand biologists after comprehensive neurological examination, neuropsychological 
assessment, brain imaging and CSF biomarkers analysis, according to current diagnostic criteria(25-29). All AD patients met the NIA-AA research framework criteria and displayed a CSF profile on the AD continuum(26).AD-MCl patients followed Albert et al. definition of $\mathrm{MCl}$ due to $\mathrm{AD}$ (25). Non-AD MCl group comprised subjects with cognitive decline unrelated to $A D$, encompassing diagnosis of psychiatric disorders, systemic disorders or non-neurodegenerative disorders. Non-AD dementia group included patients withdementia with Lewy bodies (DLB, $n=6)$, behavioral variant frontotemporal dementia (FTD, $n$ $=9$ )and vascular dementia (VD, $n=7)$. Non-AD MCl and non-AD dementia patients had normal amyloid ratio $A \beta 42 / 40$ and normal or abnormal $p$-tau and t-tau. $N C$ included patients with subjective cognitive decline, anxiety, depression, or sleep apnea syndrome, presenting with normative or sub-normative cognitive scores, normal CSF biomarkers and an absence of cognitive decline at follow-up.

\section{CSF Biomarkers}

CSF was obtained through a lumbar puncture; the second and third milliliterswere collected and centrifuged to prevent blood contamination. The supernatant was stored at $-80^{\circ} \mathrm{C}$ until further analysis.

CSF core AD biomarkers ( $A \beta 42, A \beta 40$, p-tau and t-tau) were analyzed at the Department of Biochemistry at Lariboisiere University Hospital Paris, France, using commercially available INNOTEST ${ }^{\circledR}$ kits (Fujirebio Europe NV, Gent, Belgium). CSF profiles were analyzed according to the following cut-offs: At: $\mathrm{A} \beta 42 / \mathrm{A} \beta 40$ ratio <0.076; $\mathrm{T+}$ : $\mathrm{p}$-tau $>58 \mathrm{pg} / \mathrm{mL} ; \mathrm{N}+: \mathrm{t}-\mathrm{tau}>340 \mathrm{pg} / \mathrm{mL}(26)$. Patients were classified as $\mathrm{A} \beta-$ positive and $A \beta$-negative according to the $A \beta 42 / A \beta 40$ ratio.

CSF NRG1 concentrationwasmeasured using the Human NRG1 DuoSet ELISA kit (R\&D Systems, Minneapolis, MN) as reported in Mouton-Liger et al(24).

All the CSF synaptic markerswere assessedat the Clinical Neurochemistry Laboratory at the Sahlgrenska University Hospital (Mölndal, Sweden). CSF neurograninand CSF GAP-43 concentrations were measured using in-house developed ELISAs $(8,10)$.CSF SNAP-25concentration was measured by immunoprecipitation mass spectrometry according to a validated method $(9,11)$.

\section{Plasma NRG1 Measurement}

Blood samples were obtainedthrough venipuncture under fasting condition and collected into ethylenediaminetetraacetic acid (EDTA) tubes. Samples were centrifuged at 2000xg for 20 minutes at $4 \circ \mathrm{C}$. Plasma supernatant was collected and frozen at $-80 \circ \mathrm{C}$ until further use. Prior to analysis, samples were centrifuged at $2000 \mathrm{~g}$ for 10 minutes after thawing at room temperature. Plasma NRG1 wasassessed using the Human NRG1 DuoSet ELISA kit (R\&D Systems)in Mölndal, Sweden following the manufacturer's protocol. This assay has been shown to be highly sensitive to human NRG1 alpha-subunit with a sensitivity of $125-4,000 \mathrm{pg} / \mathrm{mL}(24,30,31)$. Plasma samples from study participants were analyzed in duplicates. Intra-plate and inter-plate coefficients of variation were respectively $5.9 \%$ and $7.4 \%$.Ten samples (7.9\% of samples total) were below the detection limit of the assays, including 4 NC, 3 AD, 2 non$A D$ dementia and one non-AD MCl other patients. For those samples, plasma NRG1 levels were 
interpolated from the standard curve or if this was not possible due to the very low signal the values were imputed to the lowest interpolated value. One outlier sample (plasma NRG1 value > mean+5SD) was excluded from analysis.

\section{Statistical Analysis}

Participants' characteristics were examined in 5groups: NC, AD-MCl patients, AD dementia, non-AD MCl and non-AD dementia. Patients were also divided in $A \beta$-positive and $A \beta$-negative groups according to their CSF $A \beta 42 / A \beta 40$ ratio using the clinically validated cut-off. Data are expressed as mean (standard deviation) for continuous variables or percentage (\%) for categorical variables. We used Kruskal Wallis test to compare age and mini-mental state examination (MMSE)scores between groups and Pearson's chi-square for sex. Fluid biomarkers levels were log-transformed prior to analysis and compared usinga one-way ANCOVAadjusted on age and sex followed by a post hoc least significant difference (LSD) test for pairwise group comparisons, adjusted for multiple comparisons (Bonferroni).Linear regression adjusted on age and sex was used to explore the association between CSF and plasma NRG1.Receiver operator curve (ROC) analysis was performed to study the accuracy of plasma NRG1 in differentiating the different groups. The association of plasma NRG1 with core AD CSF biomarkers, CSF synaptic markers and with MMSE were explored by linear regression adjusted on age and sex in the whole cohort and in regard to $A \beta$ status.

A $p$-value $(P)<0.05$ was overall considered significant.Statistical analyses were performed using SPSS IBM 27.0 (IBM, Armonk, NY), and GraphPad Prism 9 (GraphPad Software, San Diego, CA, USA).

\section{Data Availability}

The datasets analyzed during the current study are available from the corresponding authors on a reasonable request.

\section{Ethics and Consent}

This study was approved by the Bichat Hospital Ethics Committee of Paris Diderot University $\left(\mathrm{N}^{\circ} 10-037\right.$, 18/03/2010) and all the participants have given their written consent.

\section{Results}

\section{Cohort}

Demographics and biomarkers values in our cohort are reported Table 1.Detailed description of non-AD dementia patients is reportedin Supplemental Table 1and description of the cohort dichotomized by $A \beta$ ratio in Supplemental Table 2.AD-MCl,AD dementia and non-AD dementia patients were older than NC and non-AD MCl patients $(P=0.003-P<0.001)$. There was no difference regarding sex between groups $(P$ $=0.155)$. All further analysis was adjusted on age and sex, unless otherwise specified. AD-MCl and AD dementia patients displayed decreased CSF A 442/Aß40 ratio and increased $p$-tau and t-tau levels 
compared with other groups. CSF synaptic markers neurogranin, GAP-43 and SNAP-25 were significantly higher in $A D$ patients compared to $\mathrm{NC}$. AD-MCl, non- $A D \mathrm{MCl}, A D$ dementia and non-AD dementia patients had decreased MMSE compared to NC.

\section{Plasma NRG1Levels Across Groups}

Higher concentration of plasma NRG1 was found in AD dementia patients compared to NCafter adjustment on age and sex (940.3 versus $378.9 \mathrm{pg} / \mathrm{mL}, P<0.001$, Figure $1 \mathrm{~A})$. AD-MCl patients also displayed higher concentrations compared to $N C$ (707.6 versus $378.9 \mathrm{pg} / \mathrm{mL}, P=0.005)$. Non-AD dementia had higher levels compared to NC (615.5versus $378.9 \mathrm{pg} / \mathrm{mL}, P=0.014)$. Plasma NRG1 concentration did not differ significantly between $\mathrm{NC}$ and non-AD MCl patients.Participants were then dichotomizedaccording to their $A \beta$ status defined by CSF $A \beta 42 / A \beta 40$ ratio (Supplemental Table 2).A $\beta$ positivepatients displayed higherplasma NRG1 concentration than $A \beta$-negativepatients (774.0 versus $538.4 \mathrm{pg} / \mathrm{mL}, P=0.023$, Figure 1B).

\section{Plasma NRG1 Accuracy in Identifying AD}

We studied plasma NRG1 accuracy in discriminating AD patients from other diagnosis groups (Figure 1C). Plasma NRG1 showed good performance in differentiating AD patients from NC both at MCl stage (AUC $=88.3 \%, 95 \% \mathrm{Cl}: 77.2 \%-0.99 .6 \%$ )and at dementia stage (AUC =87.6\%, 95\% Cl: 76.9\%-98.2\%). When comparing AD-MCI to non-AD MClpatients, plasma NRG1 showed similar accuracy (AUC $=86.4 \%$, 95\% Cl: 74.7\%-98.3\%). However, its discriminating power was lower between AD patients and non-AD dementia patients (AUC $=69.3 \%, 95 \% \mathrm{Cl}: 55.7 \%-82.3 \%$ ).

\section{Correlation to CSF NRG1}

Plasma and CSF NRG1 concentrations correlated in the overall cohort $(\beta=0.372, P=0.0056$, adjusted on age and sex) (Figure 2A). This correlation was also detected in the $A \beta$-positive group $(\beta=0.292, P=$ 0.034). No correlation was observed between plasma and CSF NRG1 in the A $\beta$-negative group $(\beta=0.156$, $P=0.305)$.

\section{Correlation to AD Biomarkers}

Plasma NRG1 displayed a weak inverse correlation with CSF A $342 / A \beta 40$ ratio in the whole cohort ( $\beta=-0.197, P=0.043$, adjusted on age and sex, Figure 2B). This correlation was stronger in the A $\beta$-positive group ( $\beta=-0.372, P=0.003)$. CSF $p$-tau and CSF t-tau displayed a stronger correlation with plasma NRG1 in the whole population (respectively: $\beta=0.361, P<0.001$ and $\beta=0.423, P<0.001$ ) (Figure $2 \mathrm{C}-\mathrm{D}$ ). These correlations were both sustained in the $A \beta$-positive patients (CSF p-tau: $\beta=0.430, P<0.001$; CSF t-tau: $\beta=0.209, P<0.001)$. There was no correlationbetween plasma NRG1 and CSF A $\beta 42 / A \beta 40$ ratio, $p$-tau and $\mathrm{t}$-tau in $A \beta$-negativepatients.

\section{Association to Synaptic Biomarkers and to Cognition}


We studied the association of plasma NRG1 with three CSF synaptic biomarkers, neurogranin, GAP-43 and SNAP-25, after adjustment on age and sex (Figure 3). Plasma NRG1 levels were overall associated with CSF GAP-43 levels $(\beta=0.355, P<0.001)$ (Figure $3 A$ ). This association remained significant in $A \beta-$ positive patients $(\beta=0.434, P<0.001)$ but not in the A $\beta$-negative group. Similarly, plasma NRG1 levels were associated with CSF neurogranin levels, in the whole cohort $(\beta=0.278, P=0.002)$ and in the A $\beta$ positive patients $(\beta=0.322, P=0.007$ ) (Figure 3B). CSF SNAP-25 levels were associated with plasma NRG1 in the whole cohort $(\beta=0.327, P=0.001)$ as in the A $\beta$-positive $(\beta=0.375, P=0.004)$ and in A $\beta$-negative group $(\beta=0.339, P=0.026$, Figure $3 C)$.

MMSE scores were significantly associated with plasma NRG1 levels after adjustment on age and sex $(\beta=-0.188, P=0.038$, Figure 3D). This association was sustained in the $A \beta$-positive group $(\beta=-0.255$, $P=0.037)$ but not in the $A \beta$-negative group.

\section{Discussion}

Accessible biomarkers monitoring synaptic dysfunction and loss would be of great clinical use in AD for early diagnosis, prediction and monitoring of cognitive decline, and for drug evaluation. In this study, we report that plasma synaptic marker NRG1 i/ was increased in AD patients already at $\mathrm{MCl}$ stage ii/ had a promising $A U C$ to discriminate $A D$ patients both at $\mathrm{MCl}$ and dementia stage, from $\mathrm{NC}$, iii/was associated with CSF AD biomarkers in A $\beta$-positive individuals and iv/ correlated with CSF synaptic markers and v/ was inversely correlated with cognition.

NRG1 is expressed at the synapse in multiple brain regions, including those preferentially affected in AD, as hippocampus and entorhinal cortex(32-34).Post-mortem studies have reported NRG1 accumulation in neuritic plaques in association with dystrophic neurites, activated astrocytes, and microglia in human AD brains $(21,22)$. NRG1 and ErbB4 directed immunoreactivity was observed in the hippocampus located in neuronal cell bodies and dendrites (22). Interestingly, NRG1 can be measured in human fluids $(17,23,24,30,31,35,36)$. Increased levels of CSF NRG1 in AD compared with controls and with non$A D$ related cognitive decline, have been reported in the literature, including our prior work $(23,24)$. In Pankonin et al., CSF NRG1 was increased in AD patients from an early stage of the disease. More recently, in a larger cohort using the most recent AD diagnosis criteria including CSF biomarkers, we have confirmed those results(24). CSF NRG1 was significantly associated with CSF AD core biomarkers, suggesting a possible implicationin AD pathophysiological processes. Moreover,CSF NRG1 levels correlated with other CSF synaptic markers, also suggesting that NRG1 was mainly originating from the synapse.

A previous study has already reported increased levels of plasma NRG1 in AD patients, with higher levels in advanced disease(35). However, in this work, AD was clinically diagnosedwith no biomarker to confirm underlying AD pathophysiological process andcorrelation with CSF NRG1 levels was not studied.Our study brings evidence that plasma NRG1 is increased in patients with confirmed underlying amyloid 
pathology, already at $\mathrm{MCl}$ stage.It is interesting to noteas APP, at the origin of $A \beta$, and NRG1 are both cleaved by BACE1 in the brain $(18,20)$.

Plasma NRG1 levels were significantly correlated with CSF levels in the whole cohort and this association was sustained in the A $\beta$-positive patients group.The existence of extra cerebral expression of NRG1 is known but the significant correlation between plasma and CSF levels indicates that plasma level modifications substantially arise from the central nervous system (37). Thus, this flags plasma NRG1 levels as a potential surrogate for brain NRG1 modifications in AD.Correlation to CSF synaptic markers GAP-43, neurogranin and SNAP-25 in the Aß-positive patients tends to indicate that detected NRG1 changes are related to synaptic modifications.

There was a significant association between plasma NRG1 levelsand MMSE in our whole cohort as well as in the Aß-positive patients. This finding is in agreement with the previous studies in plasma and CSF again showing that NRG1 levels associate with cognition already at early stages of the disease $(23,24,35)$.

Plasma NRG1 also displayed increased levels in non-AD dementia compared to NC and its accuracy in identifying $A D$ at dementia stage was moderate. In a study on vascular dementia, plasma NRG1 levels were found to be increased and inversely correlated to cognitive severity(38).Neuropathological studies and synaptic CSF biomarkers results have highlighted the fact that synapse dysfunction is a prominent feature in $A D$ but that it is not entirely specific to it $(39,40)$. It can also be observed in non-AD dementia, although to a much lesser extentthan in $A D$, a finding in line with our results (41).

An underlying mechanistic question to this marker iswhether alterations in NRG1 levels are related to a general process of synaptic degeneration and clearance or whether these changes occur as a response, positive or negative, to the development of $A D$ pathology, or to an increase in synaptic synthesis and release.

NRG1-ErbB4 signaling is important in regulating synaptic function at both excitatory and inhibitory synapses (42). While loss of NRG1 signaling has been shown to be pejorative to synaptic transmission, excessive NRG1 activity is also associated with synaptic dysfunction resulting from alterationof LTP at glutamatergic synapses(34,43). NRG1 has also been identified as a major susceptibility gene in schizophrenia(Stefansson et al. 2002; Mei and Xiong 2008). Mutant NRG1 mice display both excitatory and inhibitory synaptic impairment and schizophrenia-like behavioral disorder(46).NRG1 function must be precisely balanced to maintain normal glutamatergic receptor functions at synapses and excitatoryinhibitory neurotransmission.In line with those findings, evidence suggests that NRG1 increase may specifically influence cognitive function and neuropathology in $A D(47-49)$. Although not formally established,the mechanism of the increase of NRG1 could beexplained by the increased levels and activity of BACE1 observed in AD (47). Yet, its beneficial or detrimental effect is not solved. In experimental works, NRG1 overexpression could rescue APP induced toxicity in primary cortical neurons(48). In an AD mouse model, NRG1 treatment prevented A $\beta$-induced impairment of long-term potentiation in hippocampal slices via its receptor ErbB4(50). Conversely, other experimental works have 
suggested a negative effect of the NRG1-ErbB4 signaling in AD. Perfusion of NRG1 in hippocampus decreased LTP in AD mice model as well as in control mice (51). Further understandings of NRG1 responseupon amyloid pathology will allow to specify the exact synaptic eventsassociated with CSF and plasma NRG1 modifications observed in AD patients.

In addition to contributing to better understanding of $A D$ mechanisms, our finding that plasma NRG1 levels could reflect synaptic impairment in $A D$ may have major practical utility. Development of blood biomarkers measuring $A \beta$, tau and neurodegeneration processes has known great advancement recently, but, to date, there is no validated blood biomarkers reflecting synaptic pathology (14). Recent studies have reported that the measure of markers of $A D$ hallmarksin plasma such as A 42 , p-tau 181, p-tau217 and $p$-231 can identify and monitor $A D$ brain pathology with high accuracy, demonstratingthat they can be used as non-invasive toolsin $A D$ diagnosis(52-54).As synaptic impairment is one of the earliestabnormal features in $A D$,already present at the preclinical phase, an accessible non-invasive synaptic marker would be of high added value for early diagnosis(12).

Moreover, synaptic markers hold important promise for monitoringthe effects of disease-modifying treatments on synaptic degeneration. Compared with CSF markers, validated blood-based synaptic AD biomarkers would provide a fast, acceptable and cost-effective method of early detection, diagnosis and follow-up as well as a screening and follow-up tool in therapeutic trials.Our work shows that plasma NRG1 levels could be one of these potential biomarkers.

\section{Limitations}

This study has several limitations. The correlation between plasma and CSF NRG1 remained moderate. Further studies will be needed to understand if this variability is related to the blood-brain barrier's passage, NRG1 metabolism in plasma, matrix effects or interaction with peripheral NRG1. In our cohort, cognition was evaluated using MMSE, a general test. Study of plasma NRG1 relation to cognition using neuropsychological assessment with testsevaluating specifically episodic memory should give more robust evidence. We could not include measurements of AD-specific blood biomarkers such as $p$-tau or $A \beta$. Finally,confirmation of our results in larger cohorts is needed, including larger samples of non-AD dementia patients. Study of plasma NRG1 at preclinical phase will also be needed to better characterize its kinetic on the whole $A D$ spectrum.

\section{Conclusion}

Our results suggest that plasma NRG1 is a novel biomarker for synaptic dysfunction and degeneration in AD. Plasma NRG1 showed a significant increase in $A D$ patients already at $\mathrm{MCl}$ stage, and correlated with biomarkers for AD pathology, as well as with established CSF biomarkers for synaptic dysfunction in AD. As a novel blood synaptic marker, plasma NRG1 may improve the diagnosis of neurodegenerative disorders and may also be useful to monitor clinical disease progression and therapeutic response in clinical trials of novel disease-modifying drug candidates. 


\section{Abbreviations}

$A D$, Alzheimer's disease; $A \beta 40$, amyloid beta 1-40; $A \beta 42$, amyloid beta 1-42; APP, amyloid precursor protein; AUC, area under the curve; BACE1, beta-site amyloid precursor protein cleaving enzyme 1; CSF, cerebrospinal fluid; erbB4, receptor tyrosine-protein kinase erbB-4; GAP-43, growth-associated protein 43; $\mathrm{MCl}$, mild cognitive impairment; MMSE, mini mental state examination; NRG1, neuregulin1; PET, Positron emission tomography; p-tau, phosphorylated tau; SNAP-25, synaptosomal associated protein 25; t-tau, total tau

\section{Declarations}

\section{Acknowledgements}

The authors wish to sincerely thank all the patients that participated in this study as well as their relatives.

\section{Authors' contributions}

Concept and design: $\mathrm{CP}, \mathrm{FML}, \mathrm{JH}$. Acquisition, analysis, and interpretation of the data: all authors.Statistical analysis: AV. Drafting the manuscript: AV, CP, FML, JH. Review \& editing: all authors. All authors have read and agreed to the published version of themanuscript.Obtained funding: $\mathrm{CP}, \mathrm{JH}, \mathrm{HZ}$, $\mathrm{KB}$.

AV had full access to all the data in the study and takes full responsibility for the integrity of the data and the accuracy of the data analysis.

\section{Competing interests}

KB has served as a consultant or at advisory boards for Abcam, Axon, Biogen, JOMDD/Shimadzu, Lilly, MagQu, Prothena, Roche Diagnostics, and Siemens Healthineers, and as data monitoring committee for Julius Clinical and Novartis. KB is a co-founder of Brain Biomarker Solutions in Gothenburg AB (BBS), which is a part of the GU Ventures. HZ has served at scientific advisory boards and/or as a consultant for Abbvie, Alector, Annexon, Artery Therapeutics, AZTherapies, CogRx, Denali, Eisai, Nervgen, Pinteon Therapeutics, Red Abbey Labs, Passage Bio, Roche, Samumed, Siemens Healthineers, Triplet Therapeutics, and Wave, has given lectures in symposia sponsored by Cellectricon, Fujirebio, Alzecure, Biogen, and Roche, and is a co-founder of Brain Biomarker Solutions in Gothenburg AB (BBS), which is a part of the GU Ventures Incubator Program.CP is a member of the International Advisory Boards of Lilly; is a consultant for Fujirebio, Alzhois, Euroimmune, Ads Neuroscience, Roche, AgenT, and Gilead; and is involved as an investigator in several clinical trials for Roche, Esai, Lilly, Biogen, Astra-Zeneca, Lundbeck, and Neuroimmune.The other authors declare no competing interests.

\section{Funding}


AV was supported by Fondation Adolphe de Rothschild, Amicale des Anciens Internes des Hôpitaux de Paris, Fondation Philipe Chatrier and Fondation Vaincre Alzheimer. FML is supported by Fondation Planiol. HZ is a Wallenberg Scholar supported by grants from the Swedish Research Council (\#201802532), the European Research Council (\#681712), Swedish State Support for Clinical Research (\#ALFGBG-720931), the Alzheimer Drug Discovery Foundation (ADDF), USA (\#201809-2016862), the AD Strategic Fund and the Alzheimer's Association (\#ADSF-21-831376-C, \#ADSF-21-831381-C and \#ADSF21-831377-C), the Olav Thon Foundation, the Erling-Persson Family Foundation, Stiftelsen för Gamla Tjänarinnor, Hjärnfonden, Sweden (\#FO2019-0228), the European Union's Horizon 2020 research and innovation programme under the Marie Skłodowska-Curie grant agreement No 860197 (MIRIADE), European Union Joint Program for Neurodegenerative Disorders (JPND2021-00694), and the UK Dementia Research Institute at UCL. KB is supported by the Swedish Research Council (\#2017-00915), the Alzheimer Drug Discovery Foundation (ADDF), USA (\#RDAPB-201809-2016615), the Swedish Alzheimer Foundation (\#AF-742881), Hjärnfonden, Sweden (\#F02017-0243), the Swedish state under the agreement between the Swedish government and the County Councils, the ALF-agreement (\#ALFGBG715986), and European Union Joint Program for Neurodegenerative Disorders (JPND2019-466-236).

\section{References}

1. Selkoe DJ. Alzheimer's disease is a synaptic failure. Science. 2002 Oct 25;298(5594):789-91.

2. Arendt T. Synaptic degeneration in Alzheimer's disease. Acta Neuropathol. 2009 Jul;118(1):167-79.

3. DeKosky ST, Scheff SW. Synapse loss in frontal cortex biopsies in Alzheimer's disease: correlation with cognitive severity. Ann Neurol. 1990 May;27(5):457-64.

4. Terry RD, Masliah E, Salmon DP, Butters N, DeTeresa R, Hill R, et al. Physical basis of cognitive alterations in Alzheimer's disease: synapse loss is the major correlate of cognitive impairment. Ann Neurol. 1991 Oct;30(4):572-80.

5. Bastin C, Bahri MA, Meyer F, Manard M, Delhaye E, Plenevaux A, et al. In vivo imaging of synaptic loss in Alzheimer's disease with [18F]UCB-H positron emission tomography. Eur J Nucl Med Mol Imaging. 2020 Feb;47(2):390-402.

6. Chen M-K, Mecca AP, Naganawa M, Finnema SJ, Toyonaga T, Lin S-F, et al. Assessing Synaptic Density in Alzheimer Disease With Synaptic Vesicle Glycoprotein 2A Positron Emission Tomographic Imaging. JAMA Neurol. 2018 Oct 1;75(10):1215-24.

7. Nilsson J, Gobom J, Sjödin S, Brinkmalm G, Ashton NJ, Svensson J, et al. Cerebrospinal fluid biomarker panel for synaptic dysfunction in Alzheimer's disease. Alzheimers Dement (Amst). 2021;13(1):e12179.

8. Portelius E, Olsson B, Höglund K, Cullen NC, Kvartsberg H, Andreasson U, et al. Cerebrospinal fluid neurogranin concentration in neurodegeneration: relation to clinical phenotypes and neuropathology. Acta Neuropathol. 2018 Sep;136(3):363-76.

9. Brinkmalm A, Brinkmalm G, Honer WG, Frölich L, Hausner L, Minthon L, et al. SNAP-25 is a promising novel cerebrospinal fluid biomarker for synapse degeneration in Alzheimer's disease. Mol 
Neurodegener. 2014 Nov 23;9:53.

10. Sandelius Å, Portelius E, Källén Å, Zetterberg H, Rot U, Olsson B, et al. Elevated CSF GAP-43 is Alzheimer's disease specific and associated with tau and amyloid pathology. Alzheimers Dement. 2019 Jan;15(1):55-64.

11. Tible M, Sandelius $\AA$, Höglund K, Brinkmalm A, Cognat E, Dumurgier J, et al. Dissection of synaptic pathways through the CSF biomarkers for predicting Alzheimer disease. Neurology. 2020 Aug 25;95(8):e953-61.

12. Milà-Alomà M, Brinkmalm A, Ashton NJ, Kvartsberg H, Shekari M, Operto G, et al. CSF Synaptic Biomarkers in the Preclinical Stage of Alzheimer Disease and Their Association With MRI and PET: A Cross-sectional Study. Neurology. 2021 Sep 23;10.1212/WNL.0000000000012853.

13. Oeckl P, Halbgebauer S, Anderl-Straub S, von Arnim CAF, Diehl-Schmid J, Froelich L, et al. Targeted Mass Spectrometry Suggests Beta-Synuclein as Synaptic Blood Marker in Alzheimer's Disease. J Proteome Res. 2020 Mar 6;19(3):1310-8.

14. Camporesi E, Nilsson J, Brinkmalm A, Becker B, Ashton NJ, Blennow K, et al. Fluid Biomarkers for Synaptic Dysfunction and Loss. Biomark Insights. 2020;15:1177271920950319.

15. Meyer D, Birchmeier C. Multiple essential functions of neuregulin in development. Nature. 1995 Nov 23;378(6555):386-90.

16. Wolpowitz D, Mason TB, Dietrich P, Mendelsohn M, Talmage DA, Role LW. Cysteine-rich domain isoforms of the neuregulin-1 gene are required for maintenance of peripheral synapses. Neuron. 2000 Jan;25(1):79-91.

17. Yau H-J, Wang H-F, Lai C, Liu F-C. Neural development of the neuregulin receptor ErbB4 in the cerebral cortex and the hippocampus: preferential expression by interneurons tangentially migrating from the ganglionic eminences. Cereb Cortex. 2003 Mar;13(3):252-64.

18. Luo X, Prior M, He W, Hu X, Tang X, Shen W, et al. Cleavage of neuregulin-1 by BACE1 or ADAM10 protein produces differential effects on myelination. J Biol Chem. 2011 Jul 8;286(27):23967-74.

19. Fleck D, van Bebber F, Colombo A, Galante C, Schwenk BM, Rabe L, et al. Dual cleavage of neuregulin 1 type III by BACE1 and ADAM17 liberates its EGF-like domain and allows paracrine signaling. J Neurosci. 2013 May 1;33(18):7856-69.

20. Kamezaki A, Sato F, Aoki K, Asakawa K, Kawakami K, Matsuzaki F, et al. Visualization of Neuregulin 1 ectodomain shedding reveals its local processing in vitro and in vivo. Sci Rep. 2016 Jul 1;6(1):28873.

21. Woo R-S, Lee J-H, Yu H-N, Song D-Y, Baik T-K. Expression of ErbB4 in the neurons of Alzheimer's disease brain and APP/PS1 mice, a model of Alzheimer's disease. Anat Cell Biol. 2011 Jun;44(2):116-27.

22. Chaudhury AR, Gerecke KM, Wyss JM, Morgan DG, Gordon MN, Carroll SL. Neuregulin-1 and erbB4 immunoreactivity is associated with neuritic plaques in Alzheimer disease brain and in a transgenic model of Alzheimer disease. J Neuropathol Exp Neurol. 2003 Jan;62(1):42-54.

23. Pankonin MS, Sohi J, Kamholz J, Loeb JA. Differential distribution of neuregulin in human brain and spinal fluid. Brain Res. 2009 Mar 3;1258:1-11. 
24. Mouton-Liger F, Dumurgier J, Cognat E, Hourregue C, Zetterberg H, Vanderstichele H, et al. CSF levels of the BACE1 substrate NRG1 correlate with cognition in Alzheimer's disease. Alzheimers Res Ther. 2020 Jul 20;12(1):88.

25. Albert MS, DeKosky ST, Dickson D, Dubois B, Feldman HH, Fox NC, et al. The diagnosis of mild cognitive impairment due to Alzheimer's disease: recommendations from the National Institute on Aging-Alzheimer's Association workgroups on diagnostic guidelines for Alzheimer's disease. Alzheimers Dement. 2011 May;7(3):270-9.

26. Jack CR, Bennett DA, Blennow K, Carrillo MC, Dunn B, Haeberlein SB, et al. NIA-AA Research Framework: Toward a biological definition of Alzheimer's disease. Alzheimers Dement. 2018;14(4):535-62.

27. Rascovsky K, Hodges JR, Knopman D, Mendez MF, Kramer JH, Neuhaus J, et al. Sensitivity of revised diagnostic criteria for the behavioural variant of frontotemporal dementia. Brain. 2011 Sep;134(Pt 9):2456-77.

28. McKeith IG, Ferman TJ, Thomas AJ, Blanc F, Boeve BF, Fujishiro H, et al. Research criteria for the diagnosis of prodromal dementia with Lewy bodies. Neurology. 2020 Apr 28;94(17):743-55.

29. Sachdev P, Kalaria R, O’Brien J, Skoog I, Alladi S, Black SE, et al. Diagnostic criteria for vascular cognitive disorders: a VASCOG statement. Alzheimer Dis Assoc Disord. 2014 Sep;28(3):206-18.

30. Hama Y, Yabe I, Wakabayashi K, Kano T, Hirotani M, Iwakura Y, et al. Level of plasma neuregulin-1 SMDF is reduced in patients with idiopathic Parkinson's disease. Neuroscience Letters. 2015 Feb;587:17-21.

31. Wang R, Wang Y, Hu R, Chen X, Song M, Wang X. Decreased plasma levels of neureglin-1 in drug naïve patients and chronic patients with schizophrenia. Neurosci Lett. 2015 Oct 8;606:220-4.

32. Ozaki M, Tohyama K, Kishida H, Buonanno A, Yano R, Hashikawa T. Roles of neuregulin in synaptogenesis between mossy fibers and cerebellar granule cells. J Neurosci Res. 2000 Mar $1 ; 59(5): 612-23$.

33. Law AJ, Shannon Weickert C, Hyde TM, Kleinman JE, Harrison PJ. Neuregulin-1 (NRG-1) mRNA and protein in the adult human brain. Neuroscience. 2004;127(1):125-36.

34. Li B, Woo R-S, Mei L, Malinow R. ErbB4, a receptor of the schizophrenia-linked protein neuregulin-1, controls glutamatergic synapse maturation and plasticity. Neuron. 2007 May 24;54(4):583-97.

35. Chang K-A, Shin KY, Nam E, Lee Y-B, Moon C, Suh Y-H, et al. Plasma soluble neuregulin-1 as a diagnostic biomarker for Alzheimer's disease. Neurochem Int. 2016 Jul;97:1-7.

36. Duan J, Wei Y, Womer FY, Zhang X, Chang M, Zhu Y, et al. Neurobiological substrates of major psychiatry disorders: transdiagnostic associations between white matter abnormalities, neuregulin 1 and clinical manifestation. Journal of Psychiatry and Neuroscience. 2021 Sep 1;46(5):E506-15.

37. Cespedes JC, Liu M, Harbuzariu A, Nti A, Onyekaba J, Cespedes HW, et al. Neuregulin in Health and Disease. Int J Brain Disord Treat. 2018;4(1):024.

38. Wang X, Zhang F, Ma W, Feng D, Zhang J, Xu J. Increased Levels of Serum Neuregulin 1 Associated with Cognitive Impairment in Vascular Dementia. Biomed Res Int. 2020;2020:6683747. 
39. Willemse EAJ, Sieben A, Somers C, Vermeiren Y, De Roeck N, Timmers M, et al. Neurogranin as biomarker in CSF is non-specific to Alzheimer's disease dementia. Neurobiology of Aging. 2021 Dec 1;108:99-109.

40. Clare R, King VG, Wirenfeldt M, Vinters HV. Synapse Loss in Dementias. J Neurosci Res. 2010 Aug 1;88(10):2083-90.

41. Scheff SW, Neltner JH, Nelson PT. Is synaptic loss a unique hallmark of Alzheimer's disease? Biochem Pharmacol. 2014 Apr 15;88(4):517-28.

42. Mei L, Nave K-A. Neuregulin-ERBB signaling in the nervous system and neuropsychiatric diseases. Neuron. 2014 Jul 2;83(1):27-49.

43. Agarwal A, Zhang M, Trembak-Duff I, Unterbarnscheidt T, Radyushkin K, Dibaj P, et al. Dysregulated Expression of Neuregulin-1 by Cortical Pyramidal Neurons Disrupts Synaptic Plasticity. Cell Reports. 2014 Aug 21;8(4):1130-45.

44. Stefansson H, Sigurdsson E, Steinthorsdottir V, Bjornsdottir S, Sigmundsson T, Ghosh S, et al. Neuregulin 1 and Susceptibility to Schizophrenia. Am J Hum Genet. 2002 Oct;71(4):877-92.

45. Mei L, Xiong W-C. Neuregulin 1 in neural development, synaptic plasticity and schizophrenia. Nat Rev Neurosci. 2008 Jun;9(6):437-52.

46. O'Tuathaigh CMP, Babovic D, O'Sullivan GJ, Clifford JJ, Tighe O, Croke DT, et al. Phenotypic characterization of spatial cognition and social behavior in mice with "knockout" of the schizophrenia risk gene neuregulin 1. Neuroscience. 2007 Jun 15;147(1):18-27.

47. Yang L-B, Lindholm K, Yan R, Citron M, Xia W, Yang X-L, et al. Elevated beta-secretase expression and enzymatic activity detected in sporadic Alzheimer disease. Nat Med. 2003 Jan;9(1):3-4.

48. Yoo J-Y, Kim H-B, Baik T-K, Lee J-H, Woo R-S. Neuregulin 1/ErbB4/Akt signaling attenuates cytotoxicity mediated by the APP-CT31 fragment of amyloid precursor protein. Exp Mol Pathol. 2021 Mar 6;120:104622.

49. Zhang H, Zhang L, Zhou D, He X, Wang D, Pan H, et al. Ablating ErbB4 in PV neurons attenuates synaptic and cognitive deficits in an animal model of Alzheimer's disease. Neurobiol Dis. 2017 Oct;106:171-80.

50. Zhang L, Fletcher-Turner A, Marchionni MA, Apparsundaram S, Lundgren KH, Yurek DM, et al. Neurotrophic and neuroprotective effects of the neuregulin glial growth factor-2 on dopaminergic neurons in rat primary midbrain cultures. J Neurochem. 2004 Dec;91(6):1358-68.

51. Seo HJ, Park JE, Choi S-M, Kim T, Cho SH, Lee K-H, et al. Inhibitory Neural Network's Impairments at Hippocampal CA1 LTP in an Aged Transgenic Mouse Model of Alzheimer's Disease. Int J Mol Sci. 2021 Jan 12;22(2).

52. Ashton NJ, Pascoal TA, Karikari TK, Benedet AL, Lantero-Rodriguez J, Brinkmalm G, et al. Plasma ptau231: a new biomarker for incipient Alzheimer's disease pathology. Acta Neuropathol. 2021 May;141(5):709-24.

53. Karikari TK, Pascoal TA, Ashton NJ, Janelidze S, Benedet AL, Rodriguez JL, et al. Blood phosphorylated tau 181 as a biomarker for Alzheimer's disease: a diagnostic performance and 
prediction modelling study using data from four prospective cohorts. Lancet Neurol. 2020;19(5):422-33.

54. Ashton N, Janelidze S, Khleifat AA, Leuzy A, Ende E van der, Karikari T, et al. Diagnostic value of plasma neurofilament light: A multicentre validation study [Internet]. In Review; 2020 Aug [cited 2021 May 19]. Available from: https://www.researchsquare.com/article/rs-63386/v1

\section{Tables}

Table 1, Demographics and biomarkers values 


\begin{tabular}{|c|c|c|c|c|c|c|}
\hline$n=127$ & $\begin{array}{l}\text { Neurologicalcontrols } \\
n=20\end{array}$ & $\begin{array}{l}\text { Non-AD } \\
\text { MCl } \\
n=19\end{array}$ & $\begin{array}{l}\text { AD-MCI } \\
n=25\end{array}$ & $\begin{array}{l}\text { ADdementia } \\
n=37\end{array}$ & $\begin{array}{l}\begin{array}{l}\text { Non-AD } \\
\text { dementia }\end{array} \\
n=26\end{array}$ & $\begin{array}{l}P- \\
\text { value }\end{array}$ \\
\hline Age, years & $60.6(9.6)$ & $\begin{array}{l}61.1 \\
(8.4)\end{array}$ & $\begin{array}{l}70.3 \\
(5.8)^{\#}\end{array}$ & $67.7(7.9)^{\#}$ & $\begin{array}{l}68.1 \\
(7.0)^{\#}\end{array}$ & $<0.001$ \\
\hline Female, n (\%) & $70 \%(14)$ & $\begin{array}{l}63 \% \\
(12)\end{array}$ & $68 \%(17)$ & $62 \%(23)$ & $38 \%(10)$ & 0.155 \\
\hline LoE, years & $11.6(3.8)$ & $\begin{array}{l}9.7 \\
(2.5)\end{array}$ & $\begin{array}{l}11.0 \\
(3.9)\end{array}$ & $9.0(3.5)^{\#}$ & $\begin{array}{l}11.2 \\
(3.4)\end{array}$ & 0.050 \\
\hline MMSE & $27.42(1.6)$ & $\begin{array}{l}25.0 \\
(2.3)^{\#}\end{array}$ & $\begin{array}{l}25.1 \\
(2.4)^{\#}\end{array}$ & $18.2(4.3)^{\#}$ & $\begin{array}{l}22.8 \\
(5.4)^{\#}\end{array}$ & $<0.001$ \\
\hline \multicolumn{7}{|l|}{$\begin{array}{l}\text { CSF } \\
\text { biomarkers }\end{array}$} \\
\hline $\begin{array}{l}\text { CSF A } \beta 42, \\
\mathrm{pg} / \mathrm{mL}\end{array}$ & $1041.6(264.4)$ & $\begin{array}{l}987.2 \\
(326.0)\end{array}$ & $\begin{array}{l}516.4 \\
(122.5)^{\#}\end{array}$ & $\begin{array}{l}548.3 \\
(135.9)^{\#}\end{array}$ & $\begin{array}{l}919.3 \\
(428.7)\end{array}$ & $<0.001$ \\
\hline $\begin{array}{l}\text { CSF } \\
\text { A } 42 / A \beta 40 \\
\text { ratio }\end{array}$ & $0.129(0.045)$ & $\begin{array}{l}0.092 \\
(0.029)\end{array}$ & $\begin{array}{l}0.051 \\
(0.027)^{\#}\end{array}$ & $\begin{array}{l}0.045 \\
(0.017)^{\#}\end{array}$ & $\begin{array}{l}0.104 \\
(0.033)\end{array}$ & $<0.001$ \\
\hline $\begin{array}{l}\text { CSF p-tau, } \\
\mathrm{pg} / \mathrm{mL}\end{array}$ & 33.7 (10.7) & $\begin{array}{l}40.7 \\
(17.2)\end{array}$ & $\begin{array}{l}79.2 \\
(22.9)^{\#}\end{array}$ & $95.7(32.5)^{\#}$ & $\begin{array}{l}45.2 \\
(20.5)^{\#}\end{array}$ & $<0.001$ \\
\hline $\begin{array}{l}\text { CSF t-tau, } \\
\mathrm{pg} / \mathrm{mL}\end{array}$ & $196.0(66.7)$ & $\begin{array}{l}223.0 \\
(100.6)\end{array}$ & $\begin{array}{l}501.7 \\
(203.4)^{\#}\end{array}$ & $\begin{array}{l}703.9 \\
(285.2)^{\#}\end{array}$ & $\begin{array}{l}305.2 \\
(152.1) \#\end{array}$ & $<0.001$ \\
\hline $\begin{array}{l}\text { CSF NRG1, } \\
\mathrm{pg} / \mathrm{mL}\end{array}$ & $295.8(107.1)$ & $\begin{array}{l}324.4 \\
(137.5)\end{array}$ & $\begin{array}{l}312.8 \\
(157.8)\end{array}$ & $\begin{array}{l}403.9 \\
(155.1)^{\#}\end{array}$ & $\begin{array}{l}315.0 \\
(134.4)\end{array}$ & 0.044 \\
\hline $\begin{array}{l}\text { CSF } \\
\text { neurogranin, } \\
\mathrm{pg} / \mathrm{mL}\end{array}$ & $208.1(69.5)$ & $\begin{array}{l}213.6 \\
(84.7)\end{array}$ & $\begin{array}{l}364.4 \\
(83.1)^{\#}\end{array}$ & $\begin{array}{l}351.4(91.9) \\
\#\end{array}$ & $\begin{array}{l}230.2 \\
(107.9)\end{array}$ & $<0.001$ \\
\hline $\begin{array}{l}\text { CSF GAP-43, } \\
\mathrm{pg} / \mathrm{mL}\end{array}$ & $1677.2(616.2)$ & $\begin{array}{l}2004.8 \\
(987.9)\end{array}$ & $\begin{array}{l}3422.9 \\
(1087.9) \\
\#\end{array}$ & $\begin{array}{l}3787.6 \\
(1388.7) \#\end{array}$ & $\begin{array}{l}2348.8 \\
(1267.8)^{\#}\end{array}$ & $<0.001$ \\
\hline $\begin{array}{l}\text { CSF SNAP-25, } \\
\mathrm{pg} / \mathrm{mL}\end{array}$ & $6.7(2.2)$ & $\begin{array}{l}8.2 \\
(3.5)\end{array}$ & $\begin{array}{l}13.1 \\
(3.7)^{\#}\end{array}$ & $17.1(5.3)^{\#}$ & 7.8 (3.9) & $<0.001$ \\
\hline \multicolumn{7}{|l|}{$\begin{array}{l}\text { Plasma } \\
\text { biomarker }\end{array}$} \\
\hline $\begin{array}{l}\text { Plasma NRG1, } \\
\mathrm{pg} / \mathrm{mL}\end{array}$ & $378.9(400.7)$ & $\begin{array}{l}488.4 \\
(392.2)\end{array}$ & $\begin{array}{l}707.6 \\
(562.7)^{\#}\end{array}$ & $\begin{array}{l}940.3 \\
(737.5)^{\#}\end{array}$ & $\begin{array}{l}615.5 \\
(486.3)^{\#}\end{array}$ & $<0.001$ \\
\hline
\end{tabular}


Data shown as mean (SD) or $\mathrm{n}(\%)$, as appropriate. Kruskall-Wallis test was used to compare age between groups and Pearson's chi-square to compare sex. Fluid biomarkers levels and MMSE were compared with a one-way ANCOVA adjusted by age and sex followed by least square difference test adjusted for multiple comparisons. ${ }^{\#}<0.05$ compared to neurological controls.

Abbreviations: $A \beta 42$, amyloid-beta 42; $A \beta 40$, amyloid-beta 40; $A D$, Alzheimer's disease; $A D-M C l, M C l$ due to Alzheimer's disease; CSF, cerebrospinal fluid; GAP-43, growth-associated protein 43; LoE, level of education; $\mathrm{MCl}$, mild cognitive impairment; MMSE, Mini-Mental State Examination; NRG1, neuregulin1; ptau, phosphorylated tau; SNAP-25, synaptosomal-associated protein 25; t-tau, total tau.

\section{Figures}
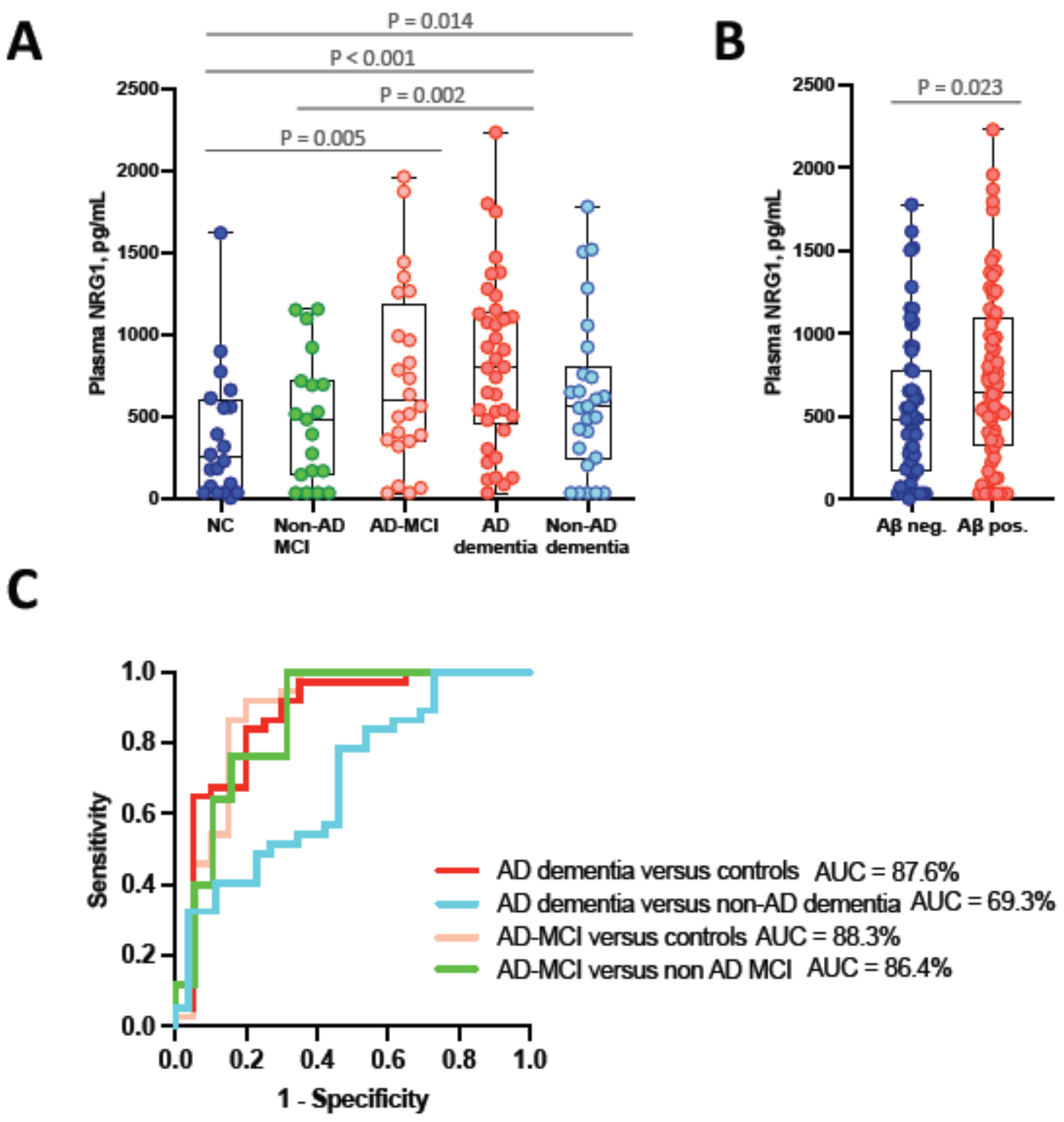

Figure 1

Plasma NRG1 levels across groups and correlation to CSF NRG1 
A, Box-plots displaying plasma NRG1 levels across diagnosis groups. Levels were compared using oneway ancova adjusted on age and sex followed by post hoc LSD test adjusted for multiple comparisons. B, Box plot displaying plasma NRG1 levels in A -positive patients $(n=62)$ and A $\beta$-negative patients $(n=55)$. Levels were compared using one-way ancova adjusted on age and sexfollowed by post hoc LSD test adjusted for multiple comparisons. C, ROC curves displaying plasma NRG1 accuracy in differentiating AD patients from neurological controls(AUC $=87.6 \%, 95 \% \mathrm{Cl}$ : 76.9\% - 98.2\%), AD dementia from non-AD dementia patients(AUC $=69.3 \%, 95 \% \mathrm{Cl}: 55.7 \%-82.3 \%), \mathrm{AD}-\mathrm{MCl}$ from NC(AUC $=88.3 \%, 95 \% \mathrm{Cl}: 77.2 \%$ $-0.99 .6 \%$ ) and $\mathrm{AD}-\mathrm{MCl}$ from non-AD MCl patients (AUC = 86.4\%, 95\% Cl: 74.7\% - 98.3\%).

Abbreviations: $A \beta$, amyloid-beta; $A D$, Alzheimer's disease; $A U C$, area under the curve; $\mathrm{MCl}$ mild cognitive impairment; LSD test, least square difference test; $\mathrm{MCl}$, mild cognitive impairment; MMSE, Mini-Mental State Examination; NRG1, neuregulin1; ROC, receiver operator characteristics.
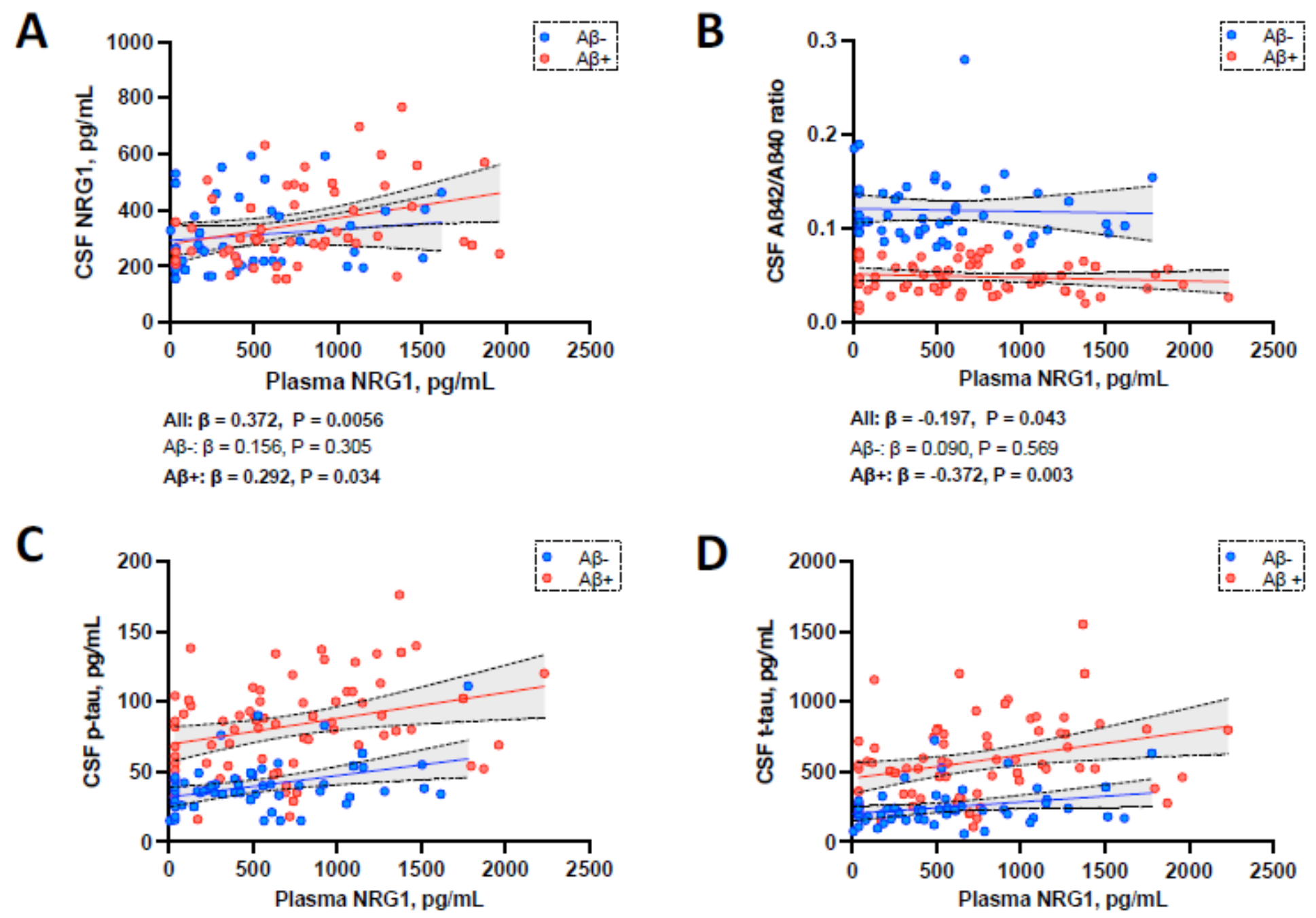

All: $\beta=0.361, P<0.001$

$A \beta-: \beta=0.248, P=0.077$

$A \beta+: \beta=0.430, P<0.001$

All: $\beta=0.423, P<0.001$
$A B-: \beta=0.249, P=0.079$
$A B+: \beta=0.209, P<0.001$

Figure 2 


\section{Association to AD CSF Biomarkers}

Association of plasma NRG1 with A,CSF NRG1 B, CSF A $342 / A \beta 40$ ratio; C, CSF p-tau; D, CSF t-tau, studied using linear regression adjusted on age and sex, in the whole cohort and in regards to $A \beta$-status. Solid lines indicate the regression line and dashed lines, the $95 \% \mathrm{Cl}$ in $\mathrm{A} \beta$-positive and $A \beta$-negative groups.

Abbreviations: $A \beta+$, amyloid-beta positive; $A \beta$-, amyloid-beta negative; $A \beta 42, \beta$-amyloid $42 ; A \beta 40, \beta$ amyloid 40; $A D$, Alzheimer's Disease; $95 \% \mathrm{Cl}$, confidence interval; CSF, cerebrospinal fluid; NRG1, neuregulin1; p-tau, phosphorylated tau; t-tau, total tau.
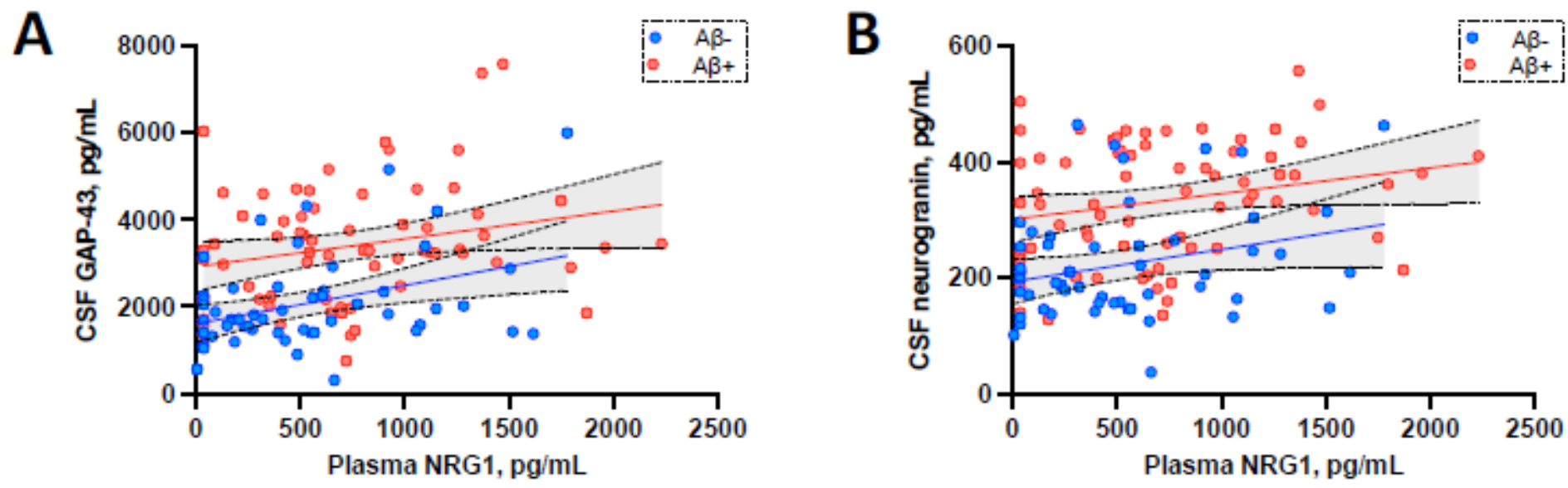

All: $\beta=0.355, P<0.001$
$A \beta-: \beta=0.274, P=0.058$
$A \beta+: \beta=0.434, P<0.001$

All: $\beta=0.278, P=0.002$

$A \beta-: \beta=0.253, P=0.088$

$A \beta+: \beta=0.322, P=0.007$

C

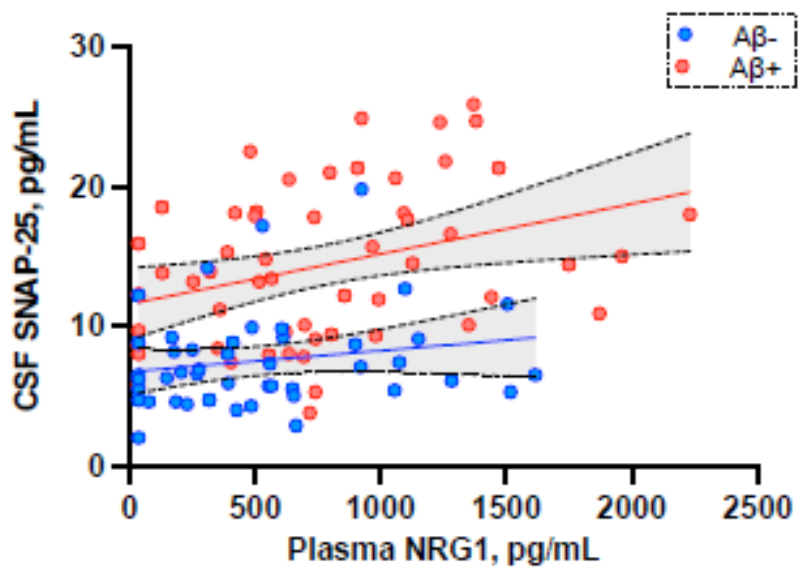

All: $\beta=0.327, P=0.001$

$A \beta-: \beta=0.339, P=0.026$

$A \beta+: \beta=0.375, P=0.004$
D

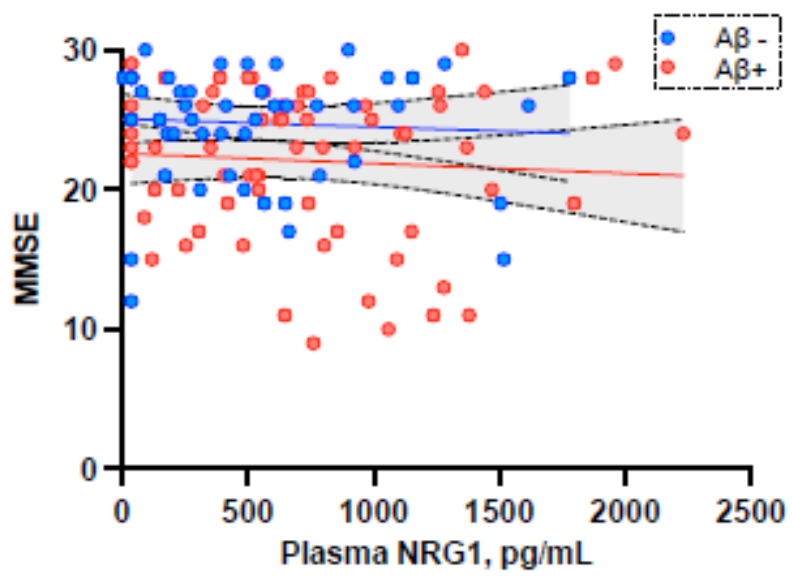

All: $\beta=-0.188, P=0.038$

$A \beta-: \beta=-0.091, P=0.519$

$A \beta+: \beta=-0.255, P=0.037$

Figure 3 
Association of plasma NRG1 with A, CSF GAP-43; B, CSF neurogranin; C, CSF SNAP-25; D, MMSE, studied using linear regression adjusted on age and sex, in the whole cohort and in regard to $A \beta$-status. Solid lines indicate the regression line and dashed lines, the $95 \% \mathrm{Cl}$ in $\mathrm{A} \beta$-positive and $A \beta$-negative groups.

Abbreviations: $A \beta+$, amyloid-beta positive; $A \beta$-, amyloid-beta negative; $95 \% \mathrm{Cl}, 95 \%$ confidence interval; $\mathrm{CSF}$, cerebrospinal fluid; GAP-43, growth-associated protein 43; MMSE, mini mental state examination; NRG1, neuregulin1; SNAP-25, synaptosomal associated protein 2

\section{Supplementary Files}

This is a list of supplementary files associated with this preprint. Click to download.

- SupplementaryData.docx 